\title{
Recours à des bactéries en tant que médicaments : traitements de l'écosystème microbien
}

\author{
Allen-Vercoe $\mathrm{E}^{1^{*}}$, Petrof $\mathrm{EO}^{2}$ \\ ${ }^{1}$ Département de la biologie moléculaire et cellulaire, Université de Guelph, Guelph, Ontario \\ ${ }^{2}$ Département de médecine, Université Queen's, Kingston (Ontario) \\ *Correspondance : eav@uoguelph.ca
}

Résumé

Dans les intestins humains réside un écosystème microbien dense et très diversifié, que l'on appelle le microbiote; ce dernier joue un rôle important dans le maintien de la santé. Les pratiques récentes de vie saine, incluant un recours généralisé à des antibiotiques, ont particulièrement affecté la diversité du microbiote. Cela compromet l'intégrité de cet écosystème vital provoquant alors une vulnérabilité aux maladies telles que l'infection au Clostridium difficile. Le traitement des patients visant à restaurer la diversité du microbiote intestinal offre une solution logique à cette infection. Bien que la bactériothérapie fécale ait commencé à gagner en importance en tant que méthode efficace permettant la restauration de la flore normale, elle n'est pas sans risque et sa mise en œuvre en clinique se heurte à de nombreux obstacles. Certains des risques et défis associés à la bactériothérapie fécale sont régulés grâce à des traitements de l'écosystème microbien, une autre approche par rapport à la bactériothérapie fécale qui se sert d'écosystèmes microbiens précis et définis pour rétablir l'équilibre et la fonctionnalité du microbiote. L'heure est venue d'utiliser les bactéries en tant que médicaments.

\section{Introduction}

Les êtres humains sont colonisés par des mille milliards de microbes; la plus petite surface de muqueuse cutanée héberge une communauté diversifiée de cellules microbiennes (1). L'intestin héberge, de loin, la plus grande densité et une diversité marquée de microbes du corps humain : le colon peut ainsi contenir jusqu'à 1012 cellules microbiennes par gramme (2). Un microbiote constitué principalement de bactéries, comprend également des archées (des procaryotes différents des bactéries), des levures et des protistes (microbes eucaryotes) (3). Bien que certains agents pathogènes opportunistes soient présents dans le microbiote, la majorité des espèces dans l'écosystème sont bénignes, même plus souvent bénéfiques, transportant de nombreuses fonctions pour le maintien d'une bonne santé. De telles fonctions incluent la modulation du système immunitaire, la production de substrats bénéfiques tels que le butyrate (qui agit en tant que source alimentaire pour les colonocytes ainsi que d'autres éléments favorisant la santé). De plus, la présence des vitamines, ainsi que la production de certaines molécules de signalisation de produits chimiques contribuent au contrôle de l'exposition pathogène à l'hôte $(4,5)$.

Malgré cette abondance de fonctions, la médecine moderne $a$, jusqu'à assez récemment, ignoré l'importance du microbiote intestinal favorisant ainsi le recours aux antibiotiques pour le traitement des infections. En plus d'encourager la résistance aux antimicrobiens, l'utilisation généralisée d'antibiotiques a diminué l'intégrité du microbiote intestinal en raison des dommages collatéraux (6). En fait, une théorie a été établie selon laquelle les dommages à l'écosystème et les pertes subséquentes en matière de diversité des espèces microbiennes ont pu contribuer à l'incidence de plusieurs maladies chroniques envahissant notre société moderne (7). Cette théorie est fondée sur de plus en plus de preuves démontrant que la diversité du microbiote intestinal est essentielle à préserver afin de prévenir des maladies inflammatoires de l'intestin 
incluant la polyarthrite psoriasique $(8,9)$. Cependant, puisque le microbiote intestinal héberge un grand nombre d'espèces microbiennes et que le profil des espèces d'une personne donnée est différent et unique (10), il est difficile de définir les mécanismes des maladies associées au microbiote.

Dans cet aperçu, nous décrivons comment l'appauvrissement de la diversité de l'écosystème peut causer des maladies prenant l'exemple de l'infection au Clostridium difficile (ICD). Nous abordons également comment les stratégies permettant de remplacer la diversité de l'écosystème grâce à des microbes fécaux peuvent être utiles dans le traitement de l'ICD. Enfin, nous démontrerons que les risques et les défis d'une telle approche peuvent être atténués par le recours à des écosystèmes microbiens bien définis.

\section{Traitement de l'infection récurrente au Clostridium difficile}

Il est bien démontré que l'ICD est le résultat de la perturbation de l'écosystème intestinal et du manque de diversité microbienne. Un problème persistant dans le milieu hospitalier, l'ICD est une complication de l'utilisation d'antibiotiques à spectre large, réduisant ainsi la diversité du microbiote intestinal de la personne hospitalisée. Sans cette diversité protectrice, l'infection au Clostridium difficile peut croître de façon incontrôlée jusqu'à atteindre de grands nombres (11). De façon similaire, aux effets des antibiotiques qui affectent la diversité microbienne intestinale, il est également bien documenté que les inhibiteurs de la pompe à protons ou la chimiothérapie peuvent contribuer au risque de développement de l'ICD (12-14). Dès qu'un seuil de croissance particulier est dépassé, l'infection au C. difficile produit une variété d'exotoxines ayant des effets nuisibles sur les colonocytes humains. Le résultat qui s'en suit est une débâcle diarrhéique pouvant mener à une colite pseudo-membraneuse, au syndrome colectasique et, pour les cas graves, au décès de la personne (11).

Actuellement, la solution thérapeutique standard à ce problème consiste à donner plus d'antibiotiques. Des médicaments tels que le métronidazole ou la vancomycine orale sont les choix agissant contre l'infection au C. difficile afin de réduire le nombre d'agents pathogènes et, par conséquent, la charge toxinogène (15). Cependant, il est important de noter une certaine particularité dans cette logique, puisque la carence même dans la diversité microbienne intestinale est directement attribuable au recours aux antibiotiques. Une utilisation soutenue d'antibiotiques peut présenter un avantage à court terme permettant de réduire temporairement le nombre d'agents pathogènes. Cependant, $C$. difficile est une bactérie productrice d'endospores pouvant sporuler lorsqu'elle est confrontée à un environnement peu propice (p. ex. en présence d'antibiotiques). Une des conséquences est la résistance des spores au métronidazole et à la vancomycine orale, permettant au $C$. difficile de germer et de créer des problèmes même lorsque l'antibiotique est éliminé (16). Parallèlement, l'exposition à l'antibiotique supprime la résistance naturelle à la colonisation du $C$. difficile - le microbiote intestinal. Les patients souffrant d'ICD se retrouvent alors dans un cycle dans lequel les antibiotiques contre $C$. difficile finissent par affecter la diversité du microbiote intestinal, empêchant le rétablissement associé à l'infection même et conduisant à une ICD récurrente. Ce constat n'est pas négligeable. Une étude récente à l'échelle des États-Unis réalisée en 2011 a estimé un demimillion de cas d'ICD pour cette année (17). L'ICD récurrente représente de $10 \%$ à $20 \%$ des cas (18).

Une solution de rechange au traitement antibiotique contre I'ICD récurrente consiste à restituer la diversité du microbiote intestinal. Le fait de rétablir cette diversité protectrice entraîne le remplacement de la souche C.difficile, un peu comme le réensemencement d'une pelouse abîmée par application d'un nouveau gazon sain qui remplacera les mauvaises herbes. La solution proposée est la « bactériothérapie fécale » qui consiste littéralement à transférer les selles d'un donneur en santé à un patient par l'intermédiaire d'un lavement rectal, d'une colonoscopie ou même d'une sonde naso-duodénale (19). Cette approche n'est pas nouvelle; les praticiens de la médecine chinoise ont utilisé des préparations de microbes fécaux pour traiter des maladies telles que la dysenterie dès le début du IV ${ }^{e}$ siècle avant J.-C. et les vétérinaires ont utilisé le principe de la transplantation fécale pour traiter les animaux souffrant de problèmes gastro-intestinaux pendant des décennies. Alors que la pratique est désagréable, elle est clairement efficace; $81 \%$ des patients ayant bénéficié d'une dose de bactériothérapie fécale pour l'ICD récurrente ont été guéris rapidement de leurs infections comparativement à $31 \%$ des patients ayant reçu de la vancomycine dans un essai clinique récemment (20). Toutefois, la bactériothérapie fécale n'est pas sans risque; bien qu'elle soit 
réalisée sous surveillance médicale, les donneurs sont rigoureusement dépistés pour déceler des agents pathogènes connus qui ont pu être transmis par les selles. II existe une telle diversité dans le microbiote intestinal humain, avec de nombreuses espèces microbiennes qui n'ont pas encore été caractérisées, qu'il est impossible aujourd'hui, de savoir si des agents pathogènes inconnus sont transmis par le biais de cette pratique.

\section{Défis relatifs à la bactériothérapie fécale}

Ainsi, bien que cette pratique soit une solution de rechange efficace à l'utilisation d'antibiotiques pour le traitement de l'ICD récurrente, elle est difficile à réglementer. Chaque selle de donneur peut être considérée comme un traitement différent et complexe. À l'heure actuelle, aucun consensus n'a été atteint sur la manière de définir et de normaliser le traitement, bien que l'utilisation de produits fécaux congelés et de « super donneurs » permettent en partie de régler le problème (19). Une autre complication est associée au fait que la supervision administrative de la procédure cautionnée par le gouvernement peut limiter sa disponibilité et risquer d'éliminer la pratique; la bactériothérapie fécale peut être réalisée au domicile, sans supervision médicale, avec du matériel qui peut être facilement obtenu dans une pharmacie. Récemment, Santé Canada a élaboré un document d'orientation intitulé Réglementation sur la bactériothérapie fécale dans le traitement des infections à $C$. difficile dans le but de mieux informer les praticiens de leur position sur cette pratique (22). Dans ce document, il est évident que Santé Canada considère le recours aux selles en tant que traitement associé à un médicament biologique puisque découlant d'une source humaine (21).

Des préoccupations sont également associées à la sécurité à long terme de la bactériothérapie fécale. Alors que pour l'ICD récurrente, les avantages à court terme sont clairs, les études sont très insuffisantes concernant les effets à long terme de ce traitement. De plus, tandis que les effets négatifs peuvent être négligeables pour les patients âgés, l'ICD devient de plus en plus courante chez les plus jeunes, chez des personnes en bonne santé qui auront besoin de ces traitements (23). Une des questions qui se pose est directement en lien avec les impératifs de respect de la sécurité avec cette thérapie qui est fort prometteuse.

\section{Traitement de l'écosystème microbien}

II est possible d'aborder les défis de la bactériothérapie fécale grâce à la création d'un « traitement de l'écosystème microbien » normalisé. Le traitement de l'écosystème microbien peut être vu comme un nouveau type de « probiotique », dans lequel des microbes bénéfiques sont d'une part choisis avec soin chez un donneur unique en bonne santé, font l'objet d'un dépistage rigoureux pour déceler tout élément négatif potentiel (p. ex. résistance aux antibiotiques) et sont d'autre part finalement recombinés dans un écosystème. Les microbes au sein des écosystèmes tendent à travailler ensemble de façon synergétique et nous formulons l'hypothèse que notre approche tire profit de cette synergie microbienne et renforce mutuellement tout effet bénéfique. Notre prototype, le MET-1 (RePOOPulate), qui s'est avéré efficace en tant que traitement de l'ICD récurrente dans un essai de démonstration du principe, a été créé en cultivant avec soin la diversité des selles d'une femme en bonne santé (24). À partir de ce bassin d'isolats, un sousensemble de 33 souches fut sélectionné; chacune d'entre elles présente une résistance minime aux antibiotiques et ne possède pas de déterminants connus de virulence; ces souches ont été formulées dans un écosystème et testées pour définir l'intégrité fonctionnelle in vitro à l'aide de la technologie du chemostat.

L'écosystème microbien défini qui en résulte présente de nombreux avantages à la bactériothérapie fécale en tant que traitement, notamment la capacité à normaliser le processus de fabrication et à créer un produit d'une qualité contrôlée. Ces derniers correspondent directement à l'une des lignes directrices clés pour l'établissement d'un règlement relatif à un médicament biologique. II est alors possible d'administrer ce produit par voie orale en tant que préparation vivante séchée à froid, ce qui simplifie significativement le traitement. Le plus grand avantage de cette approche par rapport à l'utilisation de selles réside dans le fait même que les effets à long terme du traitement peuvent être étudiés plus facilement. Ainsi, comme tout médicament biologique, il est important d'assurer la sécurité du produit avec le temps. Le MET-1 est le premier d'une série de produits de traitement de l'écosystème microbien créés pour offrir des options de traitement adaptées au style de vie du patient (p. ex. le régime alimentaire, connu pour être un facteur clé de 
la diversité de l'écosystème) (25). Alors que les obstacles réglementaires à la commercialisation de ces produits sont nombreux, les avantages sont bien identifiés et élevés. De plus, la capacité à effectuer un suivi des microbes introduits chez un patient peut contribuer à répondre aux principales questions écologiques concernant, par exemple, l'influence de l'hôte sur la fonction et la stabilité de l'écosystème. Toutefois, il est important de souligner que les travaux liés au traitement de l'écosystème microbien sont à un stade développement initial avec de nombreuses questions qui doivent être répondues. À titre d'exemple, certaines questions seraient de la nature suivante : quels sont les composants essentiels des écosystèmes visés par le traitement de l'écosystème microbien qui favorisent un avantage thérapeutique? Quelle est l'importance de la redondance fonctionnelle d'un écosystème visé par le traitement de l'écosystème microbien par rapport à son efficacité dans le traitement de la maladie en question? Les microbes introduits pendant le traitement de l'écosystème microbien colonisent-ils l'hôte de façon permanente ou agissent-ils simplement comme un « pansement » permettant à l'écosystème original de se rétablir? Le traitement de l'écosystème microbien entraîne-t-il des effets négatifs par rapport à la bactériothérapie fécale?

\section{Conclusion}

Nous disposons désormais d'une base scientifique permettant le recours aux bactéries pour des traitements thérapeutiques. La bactériothérapie fécale présente un avantage bien documenté. Cependant, elle est également confrontée à certains défis. II est clair que le traitement de l'écosystème microbien constitue un nouveau chapitre de la médecine. Dans cette nouvelle ère, les microbes doivent être considérés comme des alliés et leurs propriétés, telles que les déterminants de leur non-virulence, peuvent servir à préserver la santé.

Il est donc recommandé de recourir à l'écosystème microbien pour traiter des conditions associées à un changement dans la diversité du microbiote intestinal. À titre d'exemple, certains travaux indiquent que les approches axées sur la bactériothérapie fécale peuvent contrer l'incidence de certaines maladies telles que la colite ulcéreuse, l'obésité et le syndrome métabolique (8, 26-29).Il est nécessaire de développer des essais cliniques qui permettront de mieux éclairer la contribution de l'introduction de traitements sur mesure de l'écosystème microbien et ainsi de mieux lutter contre la dysbiose microbienne intestinale associée à certaines conditions cliniques.

\section{Conflit d'intérêts}

Les auteurs sont les cofondateurs de Nubyota LLC, une entreprise créée pour commercialiser l'application de traitements de l'écosystème microbien en médecine.

\section{Références}

(1) Human Microbiome Project C. Structure, function and diversity of the healthy human microbiome. Nature. 2012;486(7402):207-14.

(2) O'Hara AM, Shanahan F. The gut flora as a forgotten organ. EMBO Reports. 2006;7(7):688-93.

(3) Rajilic-Stojanovic M, de Vos WM. The first 1000 cultured species of the human gastrointestinal microbiota. FEMS Microbiology Reviews. 2014;38(5):996-1047.

(4) Frick JS, Autenrieth IB. The gut flora and its variety of roles in health and disease. Current Topics in Microbiology and Immunology. 2013;358:273-89.

(5) Antunes LC, McDonald JA, Schroeter K, et al. Antivirulence activity of the human gut metabolome. mBio. 2014;e01183-01114.

(6) Modi SR, Collins JJ, Relman DA. Antibiotics and the gut microbiota. The Journal of Clinical Investigation. 2014;124(10):4212-8.

(7) Blaser MJ, Falkow S. What are the consequences of the disappearing human microbiota? Nature Reviews Microbiology. 2009 Dec;7(12):887-94.

(8) Matsuoka K, Kanai T. The gut microbiota and inflammatory bowel disease. Seminars in Immunopathology. 2015;37(1):47-55.

(9) Scher JU, Ubeda C, Artacho A, et al. Decreased bacterial diversity characterizes the altered gut microbiota in patients with psoriatic arthritis, resembling dysbiosis in inflammatory bowel disease. Arthritis \& Rheumatology. 2015;67(1):128-39. 
(10) Sekirov I, Russell SL, Antunes LC, Finlay BB. Gut microbiota in health and disease. Physiological Reviews. 2010;90(3):859-904.

(11) Awad MM, Johanesen PA, Carter GP, Rose E, Lyras D. Clostridium difficile virulence factors: Insights into an anaerobic spore-forming pathogen. Gut Microbes. 2014;5(5):579-93.

(12) Freedberg DE, Lebwohl B, Abrams JA. The impact of proton pump inhibitors on the human gastrointestinal microbiome. Clinics in Laboratory Medicine. 2014;34(4):771-85.

(13) McDonald EG, Milligan J, Frenette C, Lee TC. Continuous proton pump inhibitor therapy and the associated risk of recurrent Clostridium difficile infection. 2015; JAMA Internal Medicine. 2015 May 1;175(5):784-91.

(14) Zwielehner J, Lassl C, Hippe B, et al. Changes in human fecal microbiota due to chemotherapy analyzed by TaqMan-PCR, 454 sequencing and PCR-DGGE fingerprinting. PloS One. 2011;6(12):e28654.

(15) Soriano MM, Johnson S. Treatment of Clostridium difficile infections. Infectious Disease Clinics of North America. 2015;29(1):93-108.

(16) Barra-Carrasco J, Paredes-Sabja D. Clostridium difficile spores: A major threat to the hospital environment. Future Microbiology. 2014;9(4):475-86.

(17) Lessa FC, Mu Y, Bamberg WM, et al. Burden of Clostridium difficile infection in the United States. New Engl J Med. 2015;372(9):825-34.

(18) Surawicz CM. Clostridium difficile infection: Risk factors, diagnosis and management. Current Treatment Options in Gastroenterology. 2015;13(1):121-9.

(19) Merenstein D, El-Nachef N, Lynch SV. Fecal microbiology therapy: Promises and pitfalls. Journal of Pediatric Gastroenterology and Nutrition. 2014;59(2):157-61.

(20) van Nood E, Vrieze A, Nieuwdorp M, et al. Duodenal infusion of donor feces for recurrent Clostridium difficile. New Engl J Med. 2013;368(5):407-15.

(21) Allen-Vercoe E, Reid G, Viner N, et al. A Canadian Working Group report on fecal microbial therapy: Microbial ecosystems therapeutics. Canadian Journal of Gastoenterology. 2012;26(7):457-62.

(22) Health Canada. Guidance Document: Regulation of Fecal Microbiota Therapy for the Treatment of $C$. difficile Infections. Ottawa: Health Canada; 2015. (Disponible en français : http://www.hc-sc.gc.ca/dhpmps/consultation/biolog/fecal_microbiota-bacterio_fecale-fra.php)

(23) Gupta A, Khanna S. Community-acquired Clostridium difficile infection: An increasing public health threat. Infection and Drug Resistance. 2014;7:63-72.

(24) Petrof EO, Gloor GB, Vanner SJ, et al. Stool substitute transplant therapy for the eradication of Clostridium difficile infection: 'RePOOPulating' the gut. Microbiome. 2013;1:3.

(25) Xu Z, Knight R. Dietary effects on human gut microbiome diversity. British Journal of Nutrition. 2015;113 Suppl:S1-5.

(26) Mondot S, de Wouters T, Doré J, Lepage P. The human gut microbiome and its dysfunctions. Digestive Diseases. 2013;31(3-4):278-85.

(27) Tilg H, Moschen AR. Microbiota and diabetes: An evolving relationship. Gut. 2014;63(9):1513-21.

(28) Alang N, Kelly C. Weight gain after fecal microbiota transplantation. Open Forum Infectious Diseases. 2015;2(1):1-2.

(29) Vrieze A, van Nood E, Hollerman F, et al. Transfer of intestinal microbiota from lean donors increases insulin sensitivity in individuals with metabolic syndrome. Gastroenterology. 2012 Oct;143(4):913-6.e917. 